\title{
Fatigue life assessment for a welded detail: advantages of a local energetic approach and experimental validation
}

\author{
Luigi Mario Viespoli, Filippo Berto \\ Norwegian University of Science and Technology, Norway \\ luigimv@stud.ntnu.no,filippo.berto@ntnu.no
}

\author{
Aurelio Somà \\ Politecnico di Torino, Italy \\ aurelio.soma@polito.it
}

\begin{abstract}
In modern civil buildings, as well as in mechanical and naval constructions, welding is a widely diffused technological process. The fatigue assessment, as design or verification procedure, of a welded joint to evaluate his endurance with respect to a load spectrum is a challenging but of paramount importance procedure.

Several techniques have been proposed in the literature, constantly researching efficient, cost effective and reliable methods to predict the behavior of a complex welded structures. Some of them are part of norms and standards which any design engineer must respect.

The aim of the present work is to compare the results provided by some of the principal fatigue life assessment procedures for welded joints, focusing the attention on the stress intensity factor evaluation by the use of numerical methods. Finally the proposed numerical method has been experimentally validated.
\end{abstract}

KEYWORDS. Welding; fatigue; Strain energy density; Crack initiation; structural steel; Finite element analysis

\section{open Access}

Citation: Viespoli, L. M., Somà, A., Berto, F., Fatigue life assessment for a welded detail: advantages of a local energetic approach and experimental validation, Frattura ed Integrità Strutturale, 45 (2018) 121-134.

Received: 27.04 .2018

Accepted: 26.05.2018

Published: 01.07.2018

Copyright: (C) 2018 This is an open access article under the terms of the CC-BY 4.0, which permits unrestricted use, distribution, and reproduction in any medium, provided the original author and source are credited.

\section{INTRODUCTION}

$\mathrm{F}$ or the fatigue life evaluation of a welded detail, different procedures have been proposed [1], two of the most used are the Nominal Stress and the Hot Spot Stress approaches [2]. Their diffusion is due to the relative simplicity of the techniques, which makes them ideal in a complex structure, where is necessary to perform an assessment for a great number of weldments.

A drawback of these procedures is the lack of detailed information about the stress field in the area of the critical point, so their application is closely related to an extensive previous experimental work to assess the correct fatigue class for each detail in each size class. 
When proceeding to the static and fatigue life assessment for components characterized by sharp notches, the use of the Notch Stress Intensity Factors is a feasible approach, instead of the theoretical stress concentration factors [3,22-23] used for blunt notches, where no singularity in the stress field is present. Considered that the welded geometry constitutes, in fact, a point of intensification of the tresses, being a crack or a V-notch, it would be possible to execute the prediction resolving to the computation of the Notch Stress Intensity Factors, using a classic linear elastic fracture mechanics approach [4]. These parameters have two major drawbacks: require an accurate evaluation of the stresses [5], thus an extremely fine discretization and their critical value is not a constant, but a function of the notch opening angle [6].

A reduction in the number of degrees of freedom is allowed by the use of special elements [7] able to follow the asymptotic behavior of the stress field in the proximity of the stress intensification location, although this kind of element is not implemented in commercial codes.

Adopting the Strain Energy Density approach for the fatigue life assessment [8] provides a more general tool, which constitutes both a parameter for the critical life evaluation and for the computation a posteriori of the Notch Stress Intensity Factors [9]. These are correlated to the stress field in the proximity of the notch tip by closed-form analytical relations. Moreover, being the SED a function of the stiffness matrix and the nodal displacements, it value it very accurate even with an extremely coarse discretization [10]. This important property allows to use coarse meshes for the SED computation, thus the fatigue assessment, which would normally be unacceptable for an assessment based on the stress field. Another advantage of the SED over the NSIFs is that its dimensions are constant and its critical value does not depend on the notch opening angle.

The use of the deformation energy necessary to the crack initiation has been presented as Absorbed Specific Fracture Energy by Gillemot [11]. According to Sih [12], failure can be determined by the critical value Sc of the Strain Energy Density Factor S, being this parameter the product of the Strain Energy Denity and a critical distance from the notch or crack tip. Sih's theory has been proven fit also in the case of plasticity [13] and blunt tip [14]. Glinka and Molski [15] developed a local SED-based criterion, which fundamental point is the SED constancy around the notch tip, which is suitable to application also to sharp $\mathrm{V}$-notches in plain strain condition and small yielding [16].

The value adopted for the assessment is the mean SED [9], $\Delta \mathrm{W}$ over a small critic volume of typical, material dependent radius $\mathrm{R}$, containing the notch or crack tip. The material data used as reference for welded structural steel for the fatigue life predictions of the joints tested are shown in Fig. 6 and are the result of an extensive experimental campaign of ca. 900 tests from different details and with main plate thickness between 6 and 100mm [17-20].
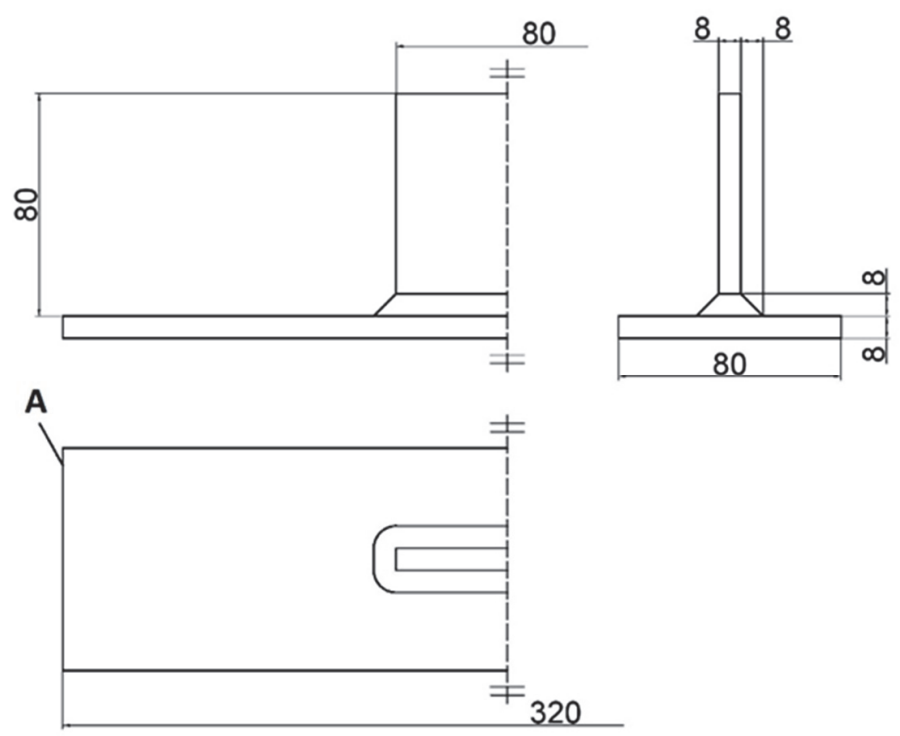

Figure 1: Geometry object of the test. The samples have been realized in S355 structural steel, welded with MIG process using ESAB OK AUTROD 12.51 copper coated wire as filling material. Dimensions in $\mathrm{mm}$.

The goal of this work is to provide a critic confront in terms of preprocessing difficulty and accuracy of the results between two of the most diffused fatigue life assessment procedures, the Nominal Stress and the Structural Hot Spot Stress approaches and the local energetic approach based on the mean Strain Energy Density. Some short notes will be reported from the literature on the topic, after which the numerical investigation and the test results are presented and confronted. 
To provide a comparison between the results provided by the different techniques, a certain geometry has been assumed as reference. That is a non-load carrying T-shaped joint, constituted by a plate and a welded longitudinal attachment. The weld fillet originates a $\mathrm{V}$-notch of opening angle $135^{\circ}$, constituting the critical point for this class of joint. The joint has been produced in six specimens and fatigue tested to longitudinal traction with load ratio $\mathrm{R}=0$. The nominal dimensions of the specimens are reported in Fig. 1.

\section{NotCH STRESS INTENSITY FACTORS AND STRAIN ENERGY DENSITY}

I

$\mathrm{t}$ is necessary, in the opinion of the authors, to present a brief overview on the analytical basis of the NSIFs and SED $[4,5,9]$. The weld toe of a joint constitutes practically a lateral open notch, which may be, according to the radius at the notch tip, sharp or blunt. The stress field is singular at the tip, with singularity exponent function of the notch opening angle. The exponents for the symmetric and the skew-symmetric stress field are, respectively, the William's eigenvalues [6] defined by:

$$
\begin{aligned}
& \sin \left(\lambda_{1} q \pi\right)+\lambda_{1} \sin (q \pi)=0 \\
& \sin \left(\lambda_{2} q \pi\right)+\lambda_{2} \sin (q \pi)=0
\end{aligned}
$$

Being q related to $2 \alpha$ by $2 \alpha=\pi(2-\mathrm{q})$.

The linearity of the problem allows to write the stress field as the sum of two components, each one defined but for two constant values a1 and a2.

The stress components for the opening mode (Mode I fracture) are:

$$
\left\{\begin{array}{c}
\sigma_{\theta} \\
\sigma_{r} \\
\tau_{r \theta}
\end{array}\right\}=\lambda_{1} r^{\lambda_{1}-1} a_{1}\left[\left\{\begin{array}{l}
\left(1+\lambda_{1}\right) \cos \left(1-\lambda_{1}\right) \theta \\
\left(3-\lambda_{1}\right) \cos \left(1-\lambda_{1}\right) \theta \\
\left(1-\lambda_{1}\right) \sin \left(1-\lambda_{1}\right) \theta
\end{array}\right\}+\chi_{1}\left(1-\lambda_{1}\right)\left\{\begin{array}{c}
\cos \left(1+\lambda_{1}\right) \theta \\
-\cos \left(1+\lambda_{1}\right) \theta \\
\sin \left(1+\lambda_{1}\right) \theta
\end{array}\right\}\right]
$$

And those for the sliding mode (Mode II fracture) are:

$$
\left\{\begin{array}{c}
\sigma_{\theta} \\
\sigma_{r} \\
\tau_{r \theta}
\end{array}\right\}=\lambda_{2} r^{\lambda_{2}-1} a_{2}\left[\left\{\begin{array}{l}
\left(1+\lambda_{2}\right) \sin \left(1-\lambda_{2}\right) \theta \\
\left(3-\lambda_{2}\right) \sin \left(1-\lambda_{2}\right) \theta \\
\left(1-\lambda_{2}\right) \cos \left(1-\lambda_{2}\right) \theta
\end{array}\right\}+\chi_{2}\left(1-\lambda_{2}\right)\left\{\begin{array}{c}
\sin \left(1+\lambda_{2}\right) \theta \\
-\sin \left(1+\lambda_{2}\right) \theta \\
\cos \left(1+\lambda_{2}\right) \theta
\end{array}\right\}\right]
$$

being:

$$
\chi_{i}=-\frac{\sin \left[\left(1-\lambda_{1}\right) q \pi / 2\right]}{\sin \left[\left(1-\lambda_{i}\right) q \pi / 2\right]}
$$

where $i$ is 1 or 2 depending on the mode considered.

The symmetric and skew-symmetric components are uncoupled along the notch bisector direction $\theta=0$, with the shear component $\tau \mathrm{r} \theta$ depending only on the sliding mode while the $\sigma \mathrm{r}$ and $\sigma \theta$ components depend only on the opening mode. Along this particular direction it is possible to extend the idea of Stress Intensity Factor to open notches, originating the sequent definition for Notch-Stress Intensity Factor:

$$
\begin{aligned}
& K_{1}=\sqrt{2 \pi} \lim _{r \rightarrow 0}\left(\sigma_{\theta}\right)_{\theta=0} r^{1-\lambda_{1}} \\
& K_{2}=\sqrt{2 \pi} \lim _{r \rightarrow 0}\left(\tau_{r \theta}\right)_{\theta=0} r^{1-\lambda_{2}}
\end{aligned}
$$


The constant value $\sqrt{2 \pi}$ is added so that, in case of opening angle $2 \alpha=0, K_{1}=K_{I}$ and $K_{2}=K_{I I}$.

As discussed, the use of the NSIFs for the fatigue life assessment of a notched component present two main difficulties: the critical value dependent on the notch opening angle and the necessity of a very refined mesh to reproduce the asymptotic trend of the stress field. If considering the real behavior of the material, characterized by plasticity, the predictions based on the stress evaluation tend to underestimate the service life of the component. According to this consideration, the critical value influencing the fatigue life is not anymore the peak stress, but an average value on a small but finite domain surrounding the notch or crack tip. Known that the Strain Energy Density can be related to the crack initiation life, the value to be considered is also in this case not a local peak, tending as well asymptotically to infinite, but its average on a small finite value around the stress singularity point. A fundamental step in this assessment procedure is defining the size of this critical volume. The following passages report recall the fundamental steps to formulate the energetic approach.

For an isotropic linear elastic material, the strain energy density (SED) is expressed by the law:

$$
W(r, \theta)=\frac{1}{2 E}\left\{\sigma_{11}^{2}+\sigma_{22}^{2}+\sigma_{33}^{2}-2 v\left(\sigma_{11} \sigma_{22}+\sigma_{11} \sigma_{33}+\sigma_{22} \sigma_{33}\right)+2(1+v) \sigma_{12}^{2}\right\}
$$

Recalling the formulation for the symmetric and skew-symmetric stress field previously introduced on the basis of the NSIFs:

$$
\left\{\begin{array}{c}
\sigma_{\theta} \\
\sigma_{r} \\
\tau_{r \theta}
\end{array}\right\}=\frac{1}{\sqrt{2 \pi}} \frac{r^{\lambda_{-1}-1} K_{1}}{\left(1+\lambda_{1}\right)+\chi_{1}\left(1-\lambda_{1}\right)}\left[\left\{\begin{array}{l}
\left(1+\lambda_{1}\right) \cos \left(1-\lambda_{1}\right) \theta \\
\left(3-\lambda_{1}\right) \cos \left(1-\lambda_{1}\right) \theta \\
\left(1-\lambda_{1}\right) \sin \left(1-\lambda_{1}\right) \theta
\end{array}\right\}+\chi_{1}\left(1-\lambda_{1}\right)\left\{\begin{array}{c}
\cos \left(1+\lambda_{1}\right) \theta \\
-\cos \left(1+\lambda_{1}\right) \theta \\
\sin \left(1+\lambda_{1}\right) \theta
\end{array}\right\}\right]
$$

in the case of Mode I fracture and:

$$
\left\{\begin{array}{c}
\sigma_{\theta} \\
\sigma_{r} \\
\tau_{r \theta}
\end{array}\right\}=\frac{1}{\sqrt{2 \pi}} \frac{r^{\lambda_{-2}-1} K_{2}}{\left(1-\lambda_{2}\right)+\chi_{2}\left(1-\lambda_{2}\right)}\left[\left\{\begin{array}{c}
-\left(1+\lambda_{2}\right) \sin \left(1-\lambda_{2}\right) \theta \\
-\left(3-\lambda_{2}\right) \sin \left(1-\lambda_{2}\right) \theta \\
\left(1-\lambda_{2}\right) \cos \left(1-\lambda_{2}\right) \theta
\end{array}\right\}+\chi_{2}\left(1+\lambda_{2}\right)\left\{\begin{array}{c}
-\sin \left(1+\lambda_{2}\right) \theta \\
\sin \left(1+\lambda_{2}\right) \theta \\
\cos \left(1+\lambda_{2}\right) \theta
\end{array}\right\}\right]
$$

in the case of Mode II fracture.

Recurring to the superposition principle and deriving the angular stress function from the previous two equations it is possible to write the stress distribution around the notch tip as:

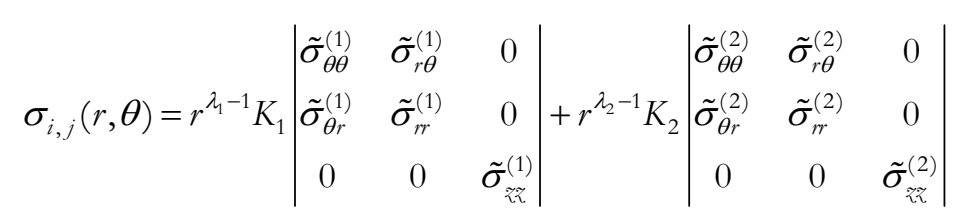

Considering that the conditions of plane stress and plane strain result, respectively, in $\sigma_{z z}=0$ and $\sigma_{z z}=v\left(\sigma_{r r}+\sigma_{\theta \theta}\right)$. The expression of the strain energy density resulting from the explicit substitution of the stresses is:

$$
W(r, \theta)=W_{1}(r, \theta)+W_{2}(r, \theta)+W_{12}(r, \theta)
$$

where:

$$
W_{1}(r, \theta)=\frac{1}{2 E} r^{2\left(\lambda_{1}-1\right)} K_{1}^{2}\left\{\tilde{\sigma}_{\theta \theta}^{(1)^{2}}+\tilde{\sigma}_{r r}^{(1)^{2}}+\tilde{\sigma}_{z z}^{(1)^{2}}-2 v\left(\tilde{\sigma}_{\theta \theta}^{(1)} \tilde{\sigma}_{r r}^{(1)}+\tilde{\sigma}_{\theta \theta}^{(1)} \tilde{\sigma}_{z z}^{(1)}+\tilde{\sigma}_{r r}^{(1)} \tilde{\sigma}_{z z}^{(1)}\right)+2(1+v) \tilde{\sigma}_{r \theta}^{(1)^{2}}\right\}
$$




$$
\begin{aligned}
W_{2}(r, \theta) & =\frac{1}{2 E} r^{2\left(\lambda_{2}-1\right)} K_{2}^{2}\left\{\tilde{\sigma}_{\theta \theta}^{(2)^{2}}+\tilde{\sigma}_{r r}^{(2)^{2}}+\tilde{\sigma}_{z z}^{(2)^{2}}-2 v\left(\tilde{\sigma}_{\theta \theta}^{(2)} \tilde{\sigma}_{r r}^{(2)}+\tilde{\sigma}_{\theta \theta}^{(2)} \tilde{\sigma}_{z z}^{(2)}+\tilde{\sigma}_{r r}^{(2)} \tilde{\sigma}_{z z}^{(2)}\right)+2(1+v) \tilde{\sigma}_{r \theta}^{(2)^{2}}\right\} \\
W_{12}(r, \theta) & =\frac{1}{E} r^{\lambda_{1}+\lambda_{2}-2} K_{1} K_{2}\left\{\tilde{\sigma}_{\theta \theta}^{(1)} \tilde{\sigma}_{\theta \theta}^{(2)}+\tilde{\sigma}_{r r}^{(1)} \tilde{\sigma}_{r r}^{(2)}+\tilde{\sigma}_{z z}^{(1)} \tilde{\sigma}_{z z}^{(2)}\right. \\
& \left.-v\left(\tilde{\sigma}_{\theta \theta}^{(1)} \tilde{\sigma}_{r r}^{(2)}+\tilde{\sigma}_{\theta \theta}^{(1)} \tilde{\sigma}_{z z}^{(2)}+\tilde{\sigma}_{r r}^{(1)} \tilde{\sigma}_{\theta \theta}^{(2)}+\tilde{\sigma}_{r r}^{(1)} \tilde{\sigma}_{z z}^{(2)}+\tilde{\sigma}_{z z}^{(1)} \tilde{\sigma}_{\theta \theta}^{(2)}+\tilde{\sigma}_{z z}^{(1)} \tilde{\sigma}_{r r}^{(2)}\right)+2(1+v) \tilde{\sigma}_{r \theta}^{(1)} \tilde{\sigma}_{r \theta}^{(2)}\right\}
\end{aligned}
$$

The integration of the strain energy density on a volume of radius $\mathrm{R}$ around the notch tip yields the elastic deformation energy:

$$
E(R)=\int_{A} W d A=\int_{0}^{R+\gamma} \int_{-\gamma}^{+\gamma}\left[W_{1}(r, \theta)+W_{2}(r, \theta)+W_{12}(r, \theta)\right] r d r d \theta
$$

being the integration field symmetric to the notch bisector, the term $W_{12}$ gives a null contribution, the resulting energy is:

$$
E(R)=E_{1}(R)+E_{2}(R)=\frac{1}{E} \frac{I_{1}(\gamma)}{4 \lambda_{1}} K_{1}^{2} R^{2 \lambda_{1}}+\frac{1}{E} \frac{I_{2}(\gamma)}{4 \lambda_{2}} K_{2}^{2} R^{2 \lambda_{2}}
$$

where:

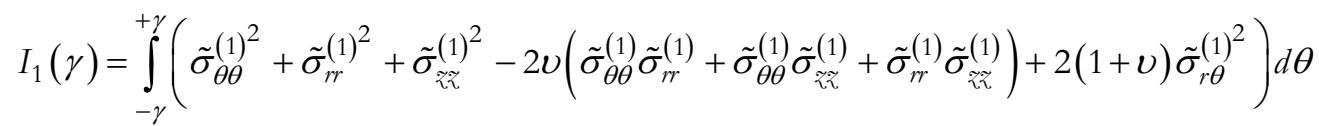

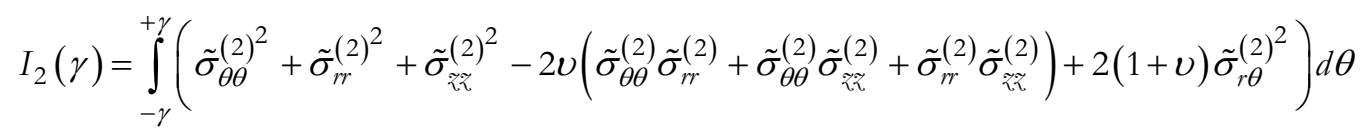

and the integration area is:

$$
A(R)=\int_{0}^{R} \int_{-\gamma}^{+\gamma} r d r d \theta=R^{2} \gamma
$$

The average of the strain energy density on the area A gives:

$$
\bar{W}=\frac{E(R)}{A(R)}=\frac{1}{E} e_{1} K_{1}^{2} R^{2\left(\lambda_{1}-1\right)}+\frac{1}{E} e_{2} K_{2}^{2} R^{2\left(\lambda_{2}-1\right)}
$$

with:

$$
\begin{aligned}
& e_{1}(2 \alpha)=\frac{I_{1}(\gamma)}{4 \lambda_{1} \gamma} \\
& e_{2}(2 \alpha)=\frac{I_{2}(\gamma)}{4 \lambda_{2} \gamma}
\end{aligned}
$$


A mean SED exceeding a critical value leads the material to failure. The condition of resistance results to be:

$$
\bar{W} \leq W_{C}
$$

In the hypothesis the material is characterised by an ideally brittle behaviour and adopting Beltrami's failure criterion, the critical value of mean SED is a function of the ultimate stress, that is:

$$
W_{C}=\sigma_{R}^{2} / 2 E
$$

The critical radius on which to compute the averaged SED is a property typical to the material and depends on its toughness. Considering a notch of zero opening angle and a null Mode II contribution due to symmetry of geometry and loading conditions, the N-SIF can be correlated to the mean SED:

$$
K_{1}=\sqrt{\frac{4 E \lambda_{1} \gamma}{I_{1}(\gamma)} \bar{W}} \mathrm{R}^{\left(1-\lambda_{1}\right)}
$$

which, at the critical conditions, becomes:

$$
K_{1}=\sqrt{\frac{2 \lambda_{1} \gamma}{I_{1}(\gamma)}} \sigma_{R} R^{\left(1-\lambda_{1}\right)}=f_{1}(2 \alpha) \sigma_{R} R^{\left(1-\lambda_{1}\right)}
$$

being $f_{1}$ a function of the opening angle.

Noting that, when the V-shaped notch opening angle is null, the notch constitutes a crack and the N-SIF coincides with the toughness of the material for LEFM.

$$
K_{1 C}\left(0^{\circ}\right)=K_{I C}=f_{1}\left(0^{\circ}\right) \sigma_{R} R^{0.5}
$$

So, the critical radius of the volume on which to compute the mean SED results:

$$
R=\left(\frac{K_{I C}}{f_{1}\left(0^{\circ}\right) \sigma_{R}}\right)^{2}
$$

\section{RAPID SED COMPUTATION IN VIRTUE OF MESH REFINEMENT INSENSITIVITY}

$\mathrm{I}$

$\mathrm{n}$ order to give a mathematical explanation to the Strain Energy Density mesh-refinement insensitivity, it is necessary to recall some of the fundamentals of the Finite Element Method [21] in linear elastic analysis. Starting from the fundamental laws in the finite element method, it is possible, with some passages [10], to express the SED stored in an element as:

$$
E_{t}=\frac{1}{2}\{d\}^{t}[K]\{d\}
$$

where:

$\{d\}$ is the nodal displacements vector and

$[K]$ is the elemental stiffness matrix. 
The mean SED is then obtained simply by dividing by the volume of the element. The key point in the computation is the dependence of the energy on the displacements and the stiffness. A continuum modeled with a coarse mesh will result having higher stiffness and lower displacements. As shown in the mesh refinement analysis, these two effects are balanced and allow to have a very precise estimate of the mean SED with a reduced number of elements.

\section{NOMINAL STRESS APPROACH}

$\mathrm{F}$ or the Nominal Stress approach, the stress considered does not take in account the micro-geometric characteristics of the joint, but only its macro-geometric shape. In particular, for the case considered, the simple plain shape allows to a fast individuation of the nominal stress, being this coincident with the remote stress applied to the main plate.
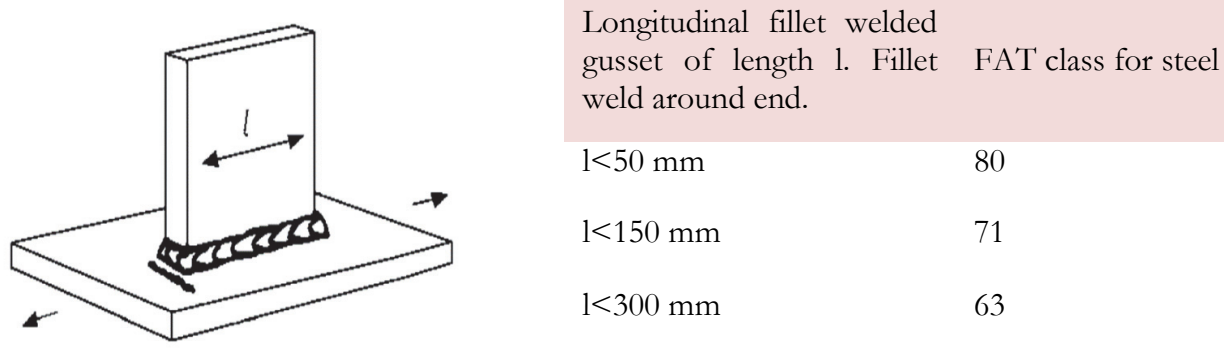

Longitudinal fillet welded gusset of length 1. Fillet FAT class for steel weld around end.

$1<50 \mathrm{~mm} \quad 80$

$1<150 \mathrm{~mm}$

$1<300 \mathrm{~mm} \quad 63$

$1>300 \mathrm{~mm} \quad 50$

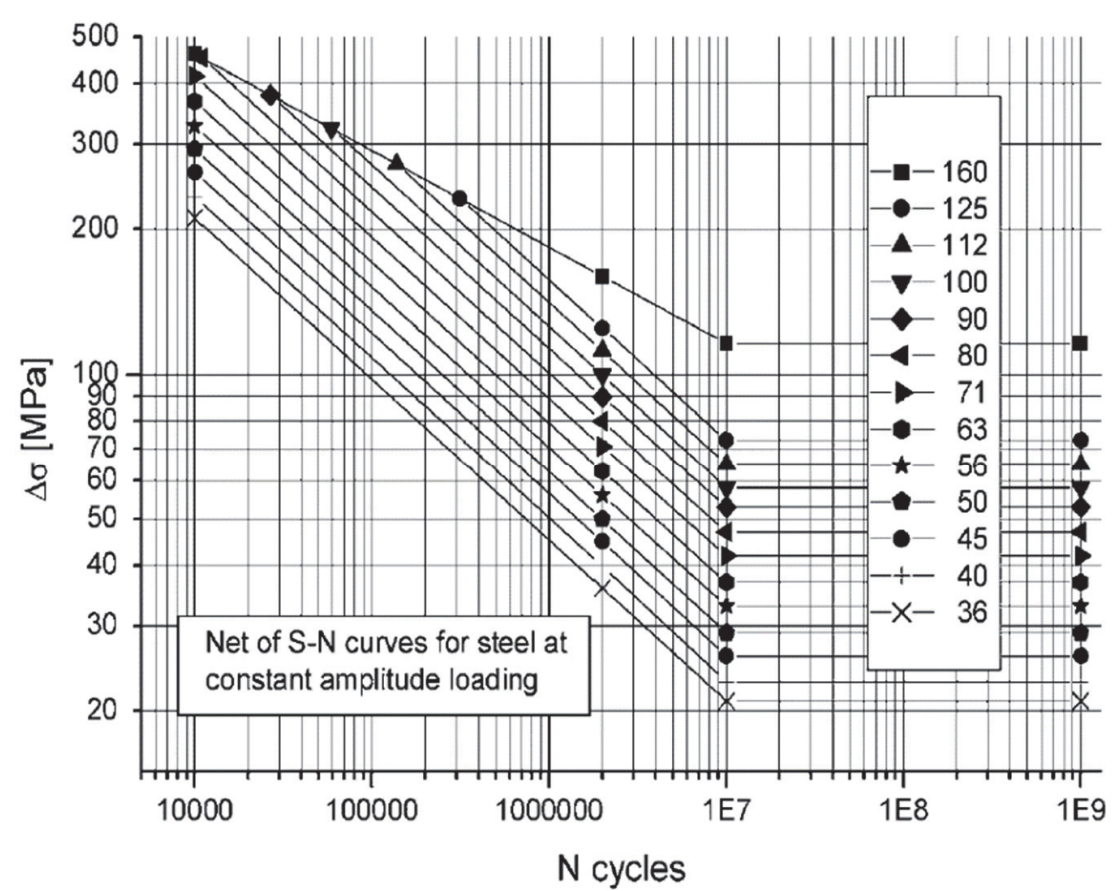

Table 1: Top: FAT classes for detail 521 page 65 IIW document depending on longitudinal attachment length [2]. Bottom: S-N fatigue curves of different fatigue classes for the fatigue life assessment of structural steel welded joints, according to the IIW document [2].

As indicated on page 65 of the IIW document, having the 1 dimension in the range between $50 \mathrm{~mm}$ and $150 \mathrm{~mm}$, the detail belongs to the fatigue class FAT 71 for steel. The estimated fatigue life for the joint subjected to a nominal stress is easily computed according to the nominal stress method as:

$$
\Delta \sigma_{r e f}^{m} N_{r e f}=\Delta \sigma_{0}^{m} N \rightarrow N
$$


where:

\begin{tabular}{ccccc}
$\Delta \sigma_{r e f}^{m}=71 \mathrm{MPa} ; N_{r e f}=2 \cdot 10^{6}$ cycles $; \quad m=3$ & & \\
\hline $\begin{array}{c}\text { Applied Load } \\
k N\end{array}$ & $\begin{array}{c}\text { Nominal Stress } \\
M P a\end{array}$ & $\begin{array}{l}\text { Expected Fatigue Life } \\
\text { Cycles }\end{array}$ & $\begin{array}{l}\text { Specimen } \\
\mathrm{N}^{\circ}\end{array}$ & $\begin{array}{l}\text { Cycles at } \\
\text { Failure }\end{array}$ \\
75 & 160 & 174761 & 1 & 235053 \\
& & & 2 & 295488 \\
65 & 140 & 260868 & 3 & 493451 \\
& & & 4 & 369923 \\
& 180 & 122740 & 5 & 154924 \\
& & & 6 & 189280 \\
\hline
\end{tabular}

Table 2: Nominal Stress Approach results summary and comparison with experimental results.

\section{STRUCTURAL Hot SpOT STRESS APPROACH}

$\mathrm{T}$

he Structural Hot Spot Stress [2,24-25] is individuated by extrapolation from the nodes (Fig. 2) at the reference points to the hot spot. Depending on whether two or three reference points are used, the extrapolation can be linear or quadratic. Moreover, depending on the stress gradient towards the weld toe, a fine mesh of linear elements or a coarse mesh of quadratic mesh can be adopted. The hot spot in question is of the type "a", that is, weld toe on the plate surface and all the three different discretization configurations introduced for the type are used in the study to compare the results. As reported in the IIW document, page 78, the class for steels for a longitudinal attachment of length inferior to $100 \mathrm{~mm}$, with fillet weld in as welded conditions, is FAT 100.

The model has been discretized with the SHELL181 element, which is a linear, four-node element with six degrees of freedom at each node.

The structural hot spot stress for linear extrapolation can be computed as:

$$
\sigma_{b s}=1.67 \sigma_{0.4 t}-0.67 \sigma_{1.0 t}
$$

The predicted fatigue life results to be:

$$
\Delta \sigma_{r f}^{m} N_{r e f}=\Delta \sigma_{0}^{m} N \rightarrow N
$$

where:

\begin{tabular}{|c|c|c|c|c|c|}
\hline Applied Load & Nominal Stress & $\begin{array}{c}\text { Structural Hot Spot } \\
\text { Stress }\end{array}$ & Expected Fatigue Life & $\begin{array}{c}\text { Specimen } \\
\mathrm{N}^{\circ}\end{array}$ & $\begin{array}{l}\text { Cycles at } \\
\text { Failure }\end{array}$ \\
\hline$k N$ & $\mathrm{MPa}$ & $\mathrm{MPa}$ & Cycles & & \\
\hline \multirow[t]{2}{*}{75} & 160 & 186 & 310807 & 1 & 235053 \\
\hline & & & & 2 & 295488 \\
\hline \multirow[t]{2}{*}{65} & 140 & 163 & 461814 & 3 & 493451 \\
\hline & & & & 4 & 369923 \\
\hline \multirow[t]{2}{*}{85} & 180 & 209 & 219074 & 5 & 154924 \\
\hline & & & & 6 & 189280 \\
\hline
\end{tabular}

$$
\Delta \sigma_{0}=\sigma_{b s} ; \Delta \sigma_{r f}^{m}=100 \mathrm{MPa} ; N_{r f}=2 \cdot 10^{6} \text { cycles } ; m=3
$$

Table 3: Structural Hot Spot Stress Approach results summary and comparison with experimental results. 


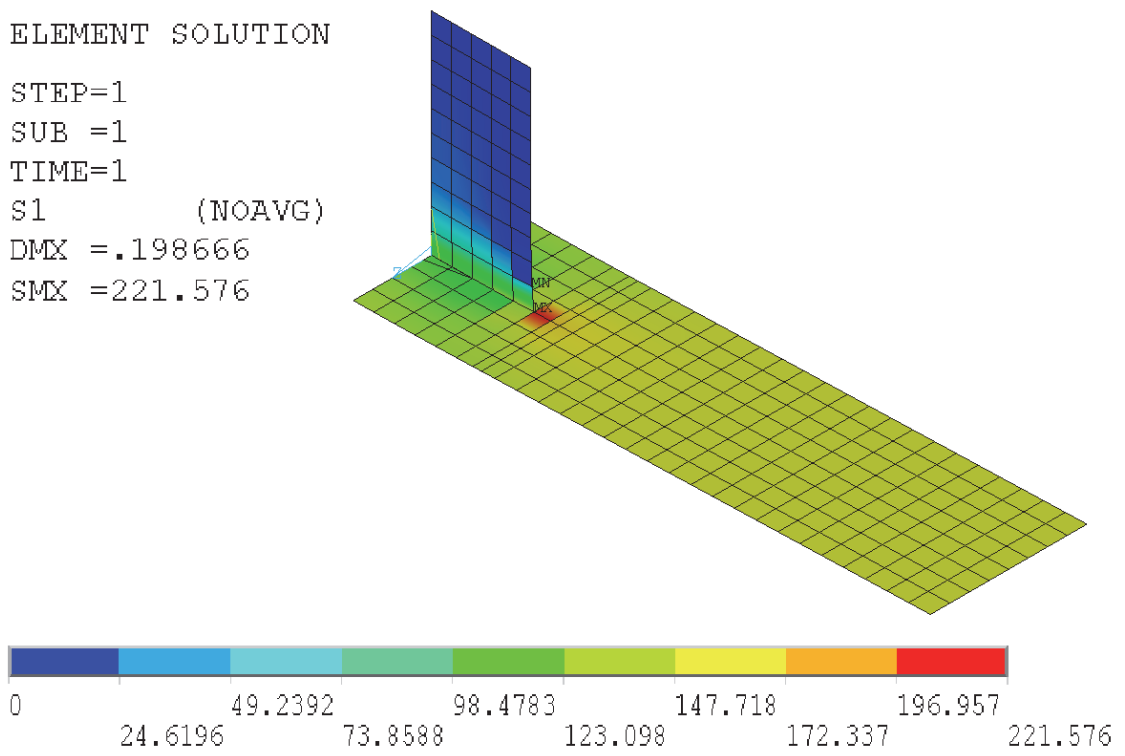

Figure 2: Coarse mapped mesh realized for the extraction of the reference stresses to be extrapolated at the weld toe as Structural Hot Spot Stress. The model is composed of 4 nodes SHELL181 elements. In figure, the first principal stress after model solution, for nominal stress of $160 \mathrm{MPa}$. In correspondence of the weld there is a clear increase in stress, but far from the singularity a 3D refined mesh could represent.

\section{STRAIN ENERGY DENSITY APPROACH}

he aim of the use of this method is to provide an improvement in the accuracy of the assessment. It combines the theory related to the study of notches in fracture mechanics with the advantage of a relatively fast solution due to the possibility of the use of a very coarse mesh. This is due to the fact that the averaged SED has very low sensibility to the refinement of the discretization.

A 3D FEM model has been realized writing an APDL code, discretizing the geometry with a coarse mesh of non-linear Solid186 elements. Taking advantage of the double symmetry of the joint, only a quarter of it has in effect been modelled, in order to reduce the resources needed for the solution. The nominal stress has then been applied on the free end of the main plate in form of a negative pressure (traction load). The control volume of radius $\mathrm{R}=0.28 \mathrm{~mm}$ is placed at the weld toe, this being the crack initiation spot for this structural detail.

The solution of the model for a unit nominal stress gives a Strain Energy on the critical volume equal to:

$$
\text { SENE }=2.01266 \cdot 10^{-7} \mathrm{Nmm}
$$

Over a volume of:

$$
\text { VOLU }=0.215503 \cdot 10^{-1} \mathrm{~mm}^{3}
$$

The resulting Averaged Strain Energy Density is:

$$
\Delta \bar{W}=\frac{S E N E}{V O L U}=9.33938 \cdot 10^{-6} \mathrm{Nmm} / \mathrm{mm}^{3}
$$

Once solved the model for a certain load, it is simple to obtain the SED for any other load imposed to the specimen, simply considering that the SED has a quadratic variation with the stress imposed. This property is used to compute the values in Tab. 5 without newly solving the model.

That is: 


$$
\frac{\Delta W}{\Delta \bar{W}}=\left(\frac{\Delta \sigma}{\Delta \bar{\sigma}}\right)^{2}
$$

which leads to the predicted fatigue life:

$$
\Delta W_{r f}^{m} N_{r f}=\Delta W^{m} N \rightarrow N
$$

where:

$$
\Delta W_{r f, 2,3 \%}^{m}=0.058 \mathrm{Nmm} / \mathrm{mm}^{3} ; N_{r f f}=2 \cdot 10^{6} \text { gycles } ; m=1.5
$$

at a probability of failure equal to $2.3 \%$.

\begin{tabular}{cc|cc}
\hline $\begin{array}{c}\text { SED for unit traction } \\
\text { load }\left[\mathrm{Nmm} / \mathrm{mm}^{3}\right]\end{array}$ & $9.33938 \cdot 10^{-6}$ & $\begin{array}{c}\text { SED for unit traction } \\
\text { load }\left[\mathrm{Nmm} / \mathrm{mm}^{3}\right]\end{array}$ & $9.15398 \cdot 10^{-6}$ \\
Number of elements & 30 & \\
Number of elements & 1920 \\
\end{tabular}

Table 4: Detail of the discretization of the critical volume of radius $\mathrm{Rc}=0,28 \mathrm{~mm}$ at the weld toe. The two different meshes, even having a very different degree of refinement, provide the same result in terms of Mean Strain Energy Density, in agreement with the SED mesh insensitivity.

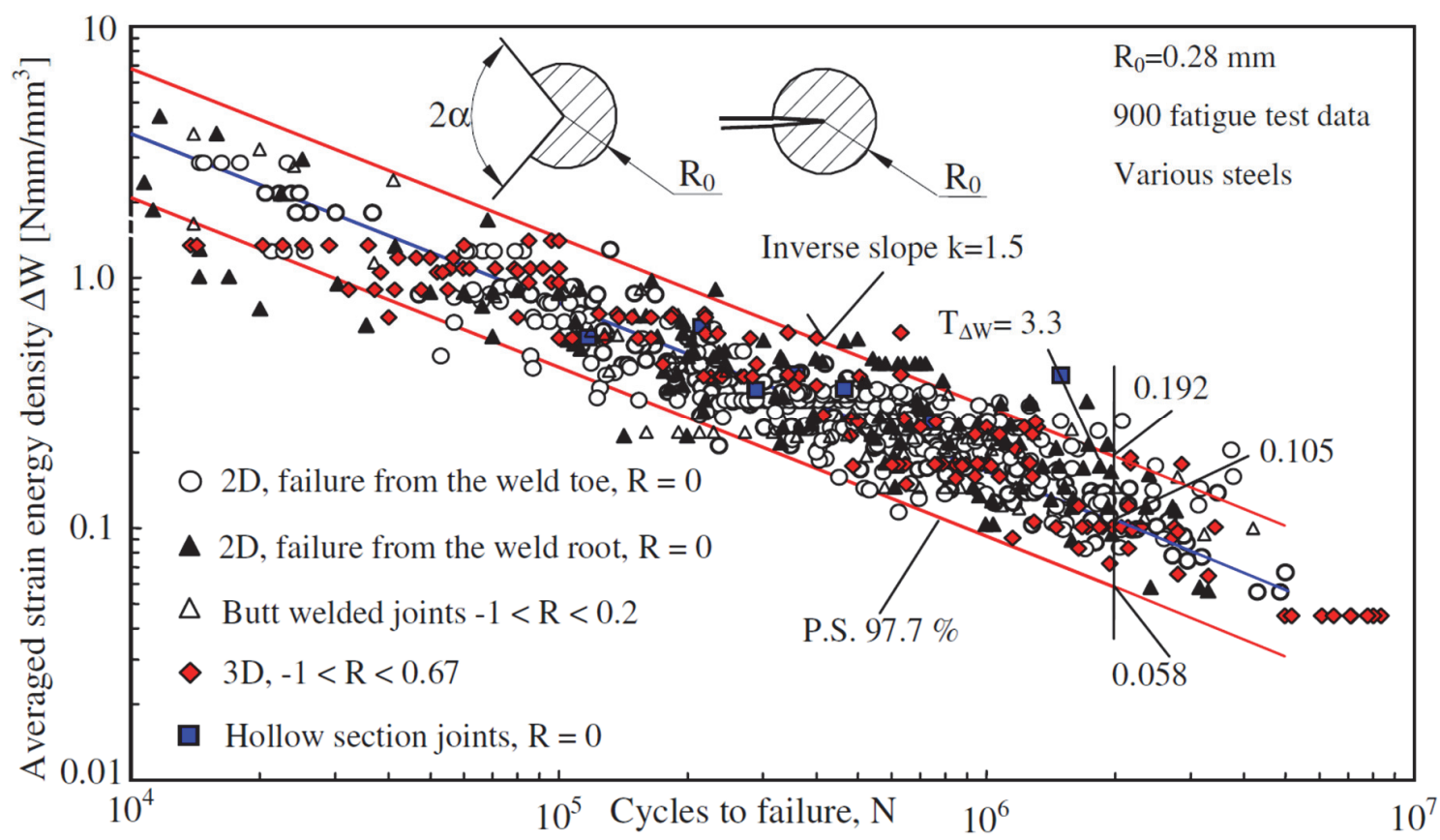

Figure 3: For the material properties related to the Strain Energy Density method, the results of a thorough test campaign already available in the literature have been used [10]. 


\begin{tabular}{|c|c|c|c|c|c|c|c|}
\hline Load & $\begin{array}{l}\text { Nominal } \\
\text { Stress }\end{array}$ & $\begin{array}{l}\text { Weld Toe } \\
\text { SED }\end{array}$ & \multicolumn{3}{|c|}{$\begin{array}{c}\text { Expected Fatigue Life in Cycles, Probability } \\
\text { of Failure }\end{array}$} & \multirow[t]{3}{*}{$\begin{array}{c}\text { Specimen } \\
\mathrm{N}^{\circ}\end{array}$} & \multirow[t]{3}{*}{$\begin{array}{l}\text { Cycles at } \\
\text { Failure }\end{array}$} \\
\hline \multirow[t]{2}{*}{$k N$} & $M P a$ & $N m m$ & $2.3 \%$ & $50 \%$ & $97.3 \%$ & & \\
\hline & & $\mathrm{mm}^{3}$ & & & & & \\
\hline \multirow[t]{2}{*}{75} & 160 & 0.239088 & 238966 & 582073 & 1439280 & 1 & 235053 \\
\hline & & & & & & 2 & 295488 \\
\hline \multirow[t]{2}{*}{65} & 140 & 0.183052 & 356706 & 868865 & 2148425 & 3 & 493451 \\
\hline & & & & & & 4 & 369923 \\
\hline \multirow[t]{2}{*}{85} & 180 & 0.302596 & 167833 & 408808 & 1010851 & 5 & 154924 \\
\hline & & & & & & 6 & 189280 \\
\hline
\end{tabular}

Table 5: Summary of the experimental results and the predicted fatigue life according to the Mean Strain Energy Density method. All the tests have been performed at a load ratio $\mathrm{R}=0$.

\section{TEST RESULTS AND COMPARISON WITH PREDICTIONS}

o verify the fitness of the models adopted to predict the fatigue life behavior of the joint, a series of tests have been executed. All the tests have been carried over on a $100 \mathrm{kN}$ Instron hydraulic traction machine at the frequency of $10 \mathrm{~Hz}$. The load ratio adopted is $\mathrm{R}=0$, then the load amplitude corresponds to the maximum load.

A total of six specimens have been realized and tested, two specimens for three load levels, as specified in Tab. 5. In the same table, are summarized also the expected fatigue lives of the specimens according to the fatigue band in Fig. 3 and the effective life of each specimen.

The specimens have been realized welding plates of S355 structural steel with MIG process, using ESAB OK AUTROD 12.51 copper coated wire as filling material and tested as welded.

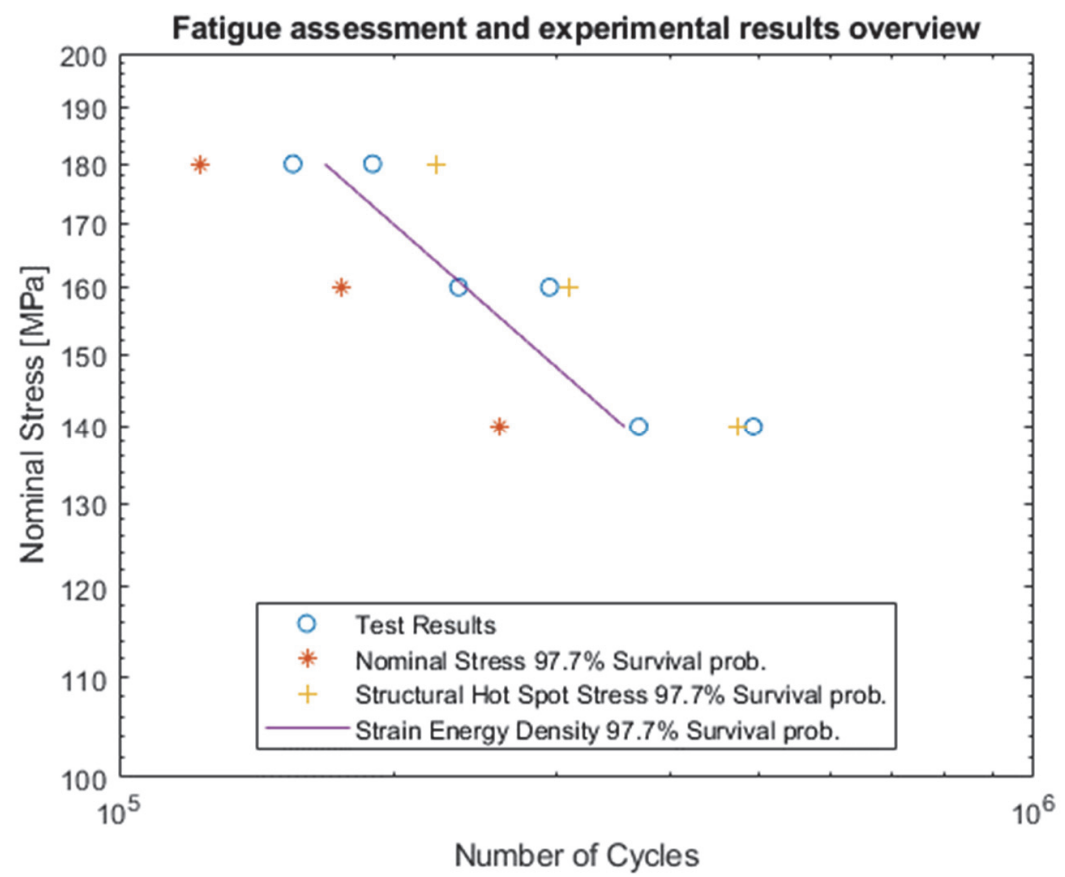

Figure 4: Overview of the fatigue life predictions for the different techniques adopted and the experimental results. 


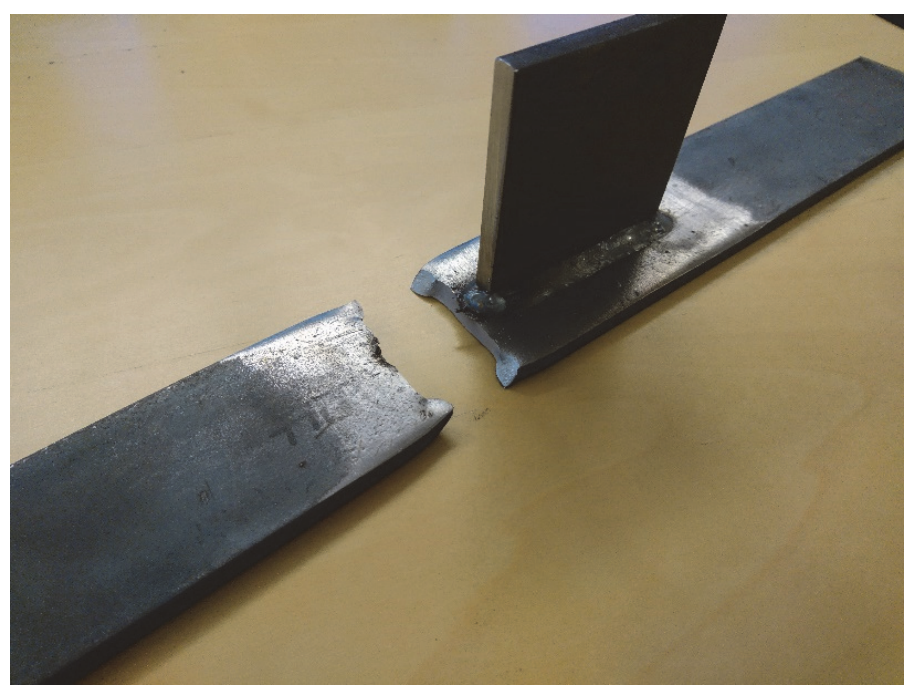

Figure 5: One of the six specimens tested, showing the fracture in the predicted location for this class of welded joints. All the specimens showed the same kind of failure.

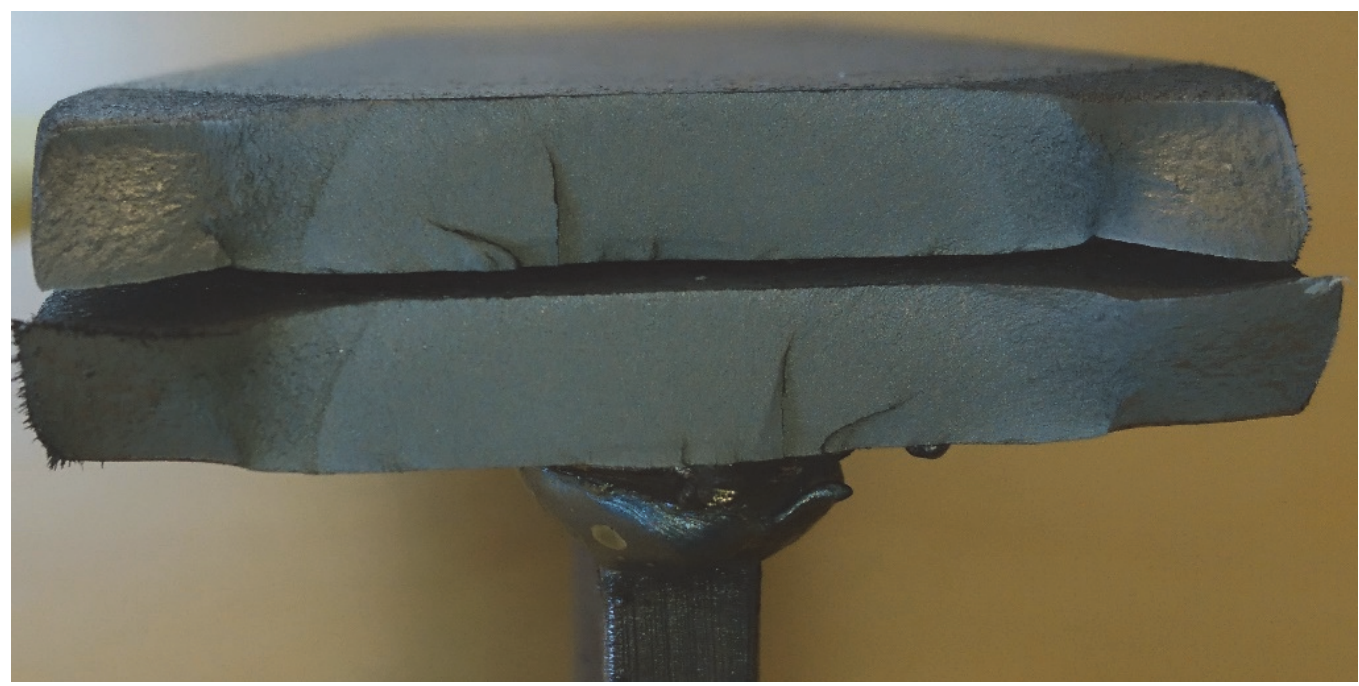

Figure 6: Detail of the fracture surface. The same type of surface characterizes all the failures in the experimental campaign.

Fig. 4 shows how the Mean Strain Energy Method is, among the implemented methods, the most precise to predict the lower extreme of the fatigue band. All the assessment are executed at a survival probability of $97.7 \%$, according to the respective fatigue bands. All the samples in the experimental campaign have shown, as expected [2], analogous kind of failure, with crack initiation and propagation from the weld toe of the main plate-longitudinal attachment conjunction. Fig. 7 represents the site of crack initiation for one of the samples. The crack starts in correspondence of the fusion line, which is not contained in a plane, but curved, thus originating the so called river pattern. The fatigue crack growths reaching the opposite side pf the main plate and propagates towards the external part of the same, being in practice a centered crack in a finite plate subjected to mode I loading. When the crack has reached a sufficient amplitude, a plastic final failure occurs. It is to be stressed how the engineering approaches followed confuse, committing an acceptable error in high cycle fatigue, the crack initiation life with the total life of the specimen. Comparing the fatigue life estimate provided by the three methods analyzed, it is clear how the SED approach predicts more accurately the lower band of the fatigue life, under the assumption of negligible propagation life, compared to the Nominal Stress and the Structural Hot Spot Stress approach. The assessment given by the Nominal Stress method appears to be conservative, but fit for the case tested, whilst the Hot Spot Stress approach has failed to safely predict the lower extreme of the fatigue band. 


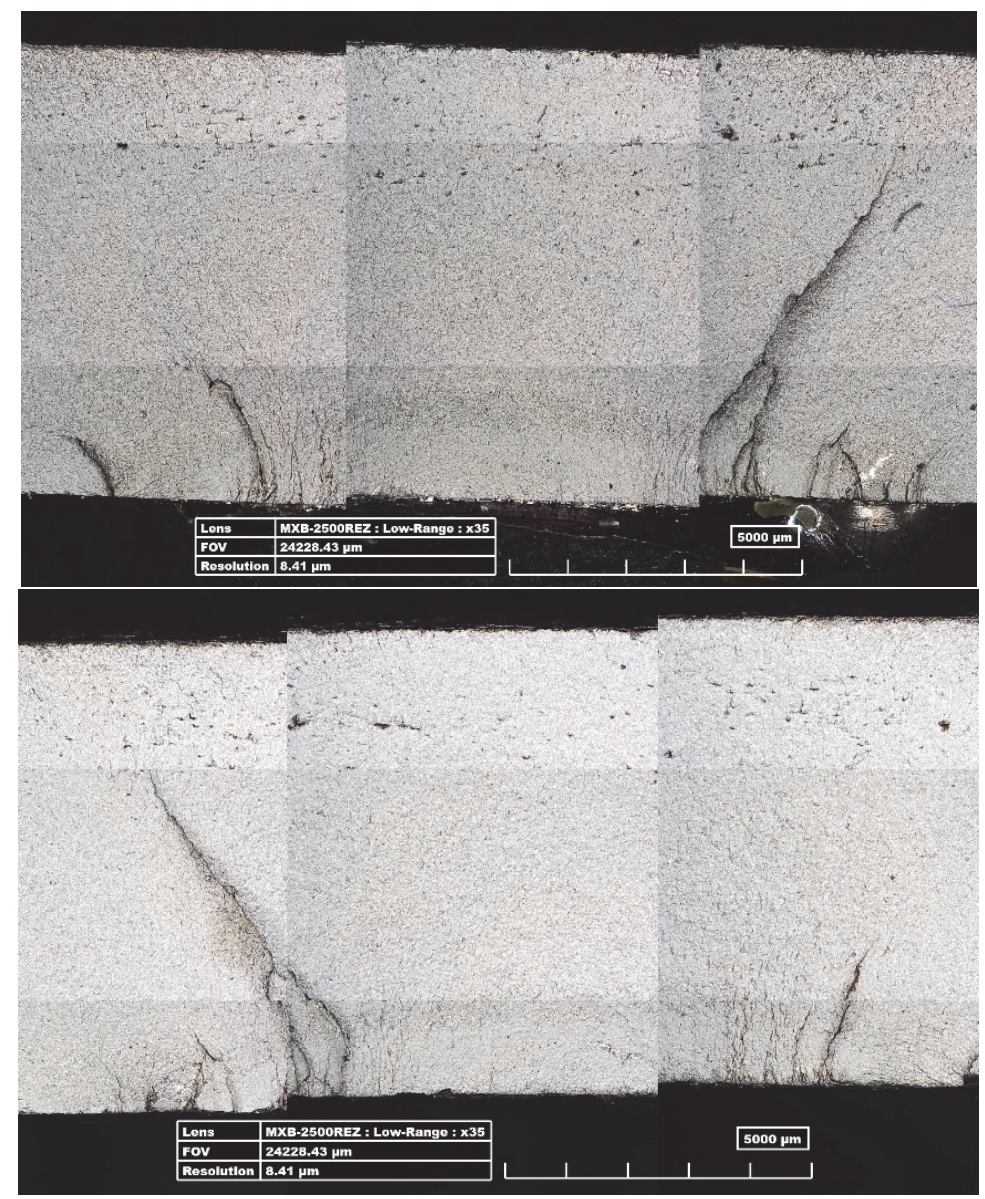

Figure 7: Capture at the optical microscope of the crack initiation area.

\section{CONCLUSIONS}

he aim of the present work was to provide a comparison among different procedures to assess the fatigue life of a welded joint. Of relevance are the Nominal Stress Method and the Hot Spot Stress Method, which nowadays are the widely used in design offices. It has been shown how these two methods can provide a fast estimate of the strength of a joint to withstand a fatigue load spectrum. The Hot Spot Stress Method is especially suitable in "heavy" carpentry, where complex structures are usually modelled through plane SHELL elements.

The drawback of the above mentioned methods is that, due to their simplicity, they do not provide detailed information on the stress field in the critic regions of the material, being such aspect demanded to the experimental procedure on which the corresponding endurable SN curves have been evaluated. The Strain Energy Density approach constitutes, instead, a more general tool, depending only on the material properties and may be adopted in a wide variety of cases for a more accurate fatigue life evaluation.

Subsequently a series of numerical analyses to compare the results provided by Nominal Stress, Hot Spot Stress and SED approaches has been performed and validated via experimental tests on a sample welded geometry. The specimens, realized with MIG welding, have been fatigue tested as welded and the failures have occurred accordingly to what stated in the literature. On the other hand, an observation of the results permits to point out that:

- $\quad$ the SED approach provides more precise results and a more reliable estimate of the fatigue life, compared to the other methods adopted;

- this advantage in design comes at the cost of a more detailed FEM modelling with respect to the Nominal and the Structural Hot Spot stress methods while, if compared to a more detailed fracture mechanics based analysis, the mesh insensitivity of the mean SED guarantees a much easier modelling. 


\section{REFERENCES}

[1] Eurocode 3: Design of steel structures - Part 1-9: Fatigue, EUROPEAN COMMITTEE FOR STANDARDIZATION Ref, No, EN 1993-1-92005: E

[2] Hobbacher, A. (2007). Recommendations for fatigue design of welded joints and components IIW document IIW1823-07

[3] Peterson, R. E. (1953). Stress Concentration Design Factors. John Wiley \& Sons ISBN 978-0471683766.

[4] Lazzarin, P. and Tovo, R. (1998). A notch intensity factor approach to the stress analysis of welds. Fatigue \& Fracture of Engineering Materials \& Structures 21, pp. 1089-1103.

[5] Lazzarin, P. and Zambardi, R. (2001). A finite-volume-energy based approach to predict the static and fatigue behavior of components with sharp V-shaped notches. International Journal of Fracture 112, pp. 275-298.

[6] Williams, M.L. (1952). Stress singularities resulting from various boundary conditions in angular corners of plates in tension. J Appl Mech 19, pp. 526-528.

[7] Henshell, R.D. and Shaw, K.G. (1975). Crack tip finite elements are unnecessary. Int J Numer Methods Eng 9, pp. 495-507.

[8] Berto, F. and Lazzarin, P. (2009). The volume-based Strain Energy Density approach applied to static and fatigue strength assessments of notched and welded structures, Procedia Engineering 01, pp. 155-158

[9] Lazzarin, P., Berto, F., Gomez, F. J. and Zappalorto, M. (2008). Some advantages derived from the use of the strain energy densityover a control volume in fatigue strength assessments of welded joints. International Journal of Fatigue 30, pp. 1345-1357.

[10] Lazzarin, P., Berto, F. and Zappalorto, M. (2010). Rapid calculations of notch stress intensity factors based on averaged strain energy density from coarse meshes: Theoretical bases and applications. International Journal of Fatigue 32, pp. 1559-1567.

[11] Gillemot, L. F. (1976). Criterion of crack initiation and spreading. Eng Fract Mech 8, pp. 239-253.

[12] Sih, G. C. (1974). Strain-energy-density factor applied to mixed mode crack problems. Int J Fract 10, pp. 305-321.

[13] Sih, G. C. (1991) Mechanics of Fracture Initiation and Propagation: Surface and volume energy density applied as failure criterion, Kluwer Academic Publisher, Dordrecht.

[14] Sih, G. C. (1974). Surface layer energy and strain energy density for a blunted crack or notch, In: Prospect of Fracture Mechanics, edited by GC Sih, HC van Elst and D Broek, Noordhoff International Publishing, Leyden, 85-102.

[15] Glinka, G. and Molski, K. (1981). A method of elastic-plastic stress and strain calculation at a notch root. Mat Science Eng 50, pp. 93-100.

[16] Lazzarin, P. and Zambardi, R. (2002). The equivalent strain energy density approach re-formulated and applied to sharp V-shaped notches under localised and generalised plasticity, Fatigue Fract Engng Mater Struct 25, pp. 917-928.

[17] Lazzarin, P., Lassen, T. and Livieri, P. (2003). A Notch Stress Intensity approach applied to fatigue life predictions of welded joints with different local toe geometry, Fatigue Fract Engng Mater Struct 26, pp. 39-48.

[18] Livieri, P. and Lazzarin, P. (2005). Fatigue strength of steel and aluminium welded joints based on generalised stress intensity factors and local strain energy values. Int J Fract 133, pp. 247-278.

[19] Lazzarin, P., Berto, F. and Radaj, D. (2006). Uniform fatigue strength of butt welded joints in terms of the local strain energy density, Proc. Int. Conference Fatigue 2006, Atlanta, USA.

[20] Lazzarin, P., Livieri, P., Berto, F. and Zappalorto, M. (2008). Local strain energy density and fatigue strength of welded joints under uniaxial and multiaxial loading. Engng Fract Mech 75, pp. 1875-1889.

[21] Zienkiewicz, O.C., Taylor, R.L. and Zhu, J.Z. (2005). The Finite Element Method: Its Basis and Fundamentals, Elsevier Butterworth-Heinemann, $6^{\text {th }}$ edition.

[22] Radaj, D. (2013). Advanced Methods of Fatigue Assessment, Springer-Verlag Berlin Heidelberg.

[23] Anderson, T.L. (1995). Fracture Mechanics - Fundamentals and Applications CRC Press.

[24] Poutiainen, I., Tanskanen, P. and Marquis, G. (2004). Finite element methods for structural hot spot stress determination - a comparison of procedures, International Journal of Fatigue 26, pp. 1147-1157.

[25] Jae-Myung Lee et al., (2010). Comparison of hot spot stress evaluation methods for welded structures, Inter J Nav Archit Oc Engng 2, pp. 200-210. 